\title{
Scan-to-BIM - An Overview of the Current State of the Art and a Look Ahead
}

\author{
H. Son ${ }^{a}$, C. Kim ${ }^{a}$, and Y. Turkan ${ }^{b}$ \\ ${ }^{a}$ Department of Architectural Engineering, Chung-Ang University, South Korea \\ ${ }^{b}$ Department of Civil, Construction and Environmental Engineering, Iowa State University, United States \\ E-mail: hjson0908@cau.ac.kr, changwan@cau.ac.kr, yturkan@iastate.edu
}

\begin{abstract}
The use of Building Information Modeling (BIM) technology to support building design and construction practices is becoming the standard in the U.S. and worldwide, and various benefits of implementing BIM, such as reduced RFIs and change orders, have already been proven. However, efficient integration of BIM with facility management (FM) systems, which requires having accurate as-built BIM, has not yet been achieved. Integrated BIM-FM systems offer significant benefits to owners such as better visualization of the spaces to be maintained in a virtual environment. BIMs, specifically MEP BIMs, are reviewed on a regular basis during project coordination meetings between stakeholders, and are updated regularly during the construction phase. However, some coordination issues related to MEP components maybe realized within the BIM, but fixed in the field; and those changes are not typically reflected in the BIM. Three dimensional (3D) point clouds provide accurate and comprehensive as-built information, and are used for creating as-built BIMs. The process of creating as-built BIM from 3D point cloud data is referred to as Scan-to-BIM. Currently available offthe-shelf Scan-to-BIM software packages require significant manual user input making the entire process cumbersome and error prone. Significant amount of research has been done both in academia and in industry to automate Scan-to-BIM process. The goal of this paper is to provide a comprehensive review on current Scan-to-BIM software and their capabilities to retrieve MEP components from 3D point clouds, while identifying challenges and the look ahead for future research.
\end{abstract}

Keywords - Laser Scanning; BIM, As-built BIM, Scan-to-BIM, MEP Systems

\section{Introduction}

Building Information Modeling (BIM) is becoming the norm for designing and delivering building projects in the U.S. and worldwide. However, efficient integration of BIM with Facility Management (FM) Systems has not yet been achieved [1]. One of the main reasons is that FM systems require accurate as-built information, and accurate as-built BIM models are not typically required in the contracts as part of the project deliverables. This is mainly due to the fact that majority of the owners are not yet fully aware of the benefits BIM has to offer [2]. Moreover, it is even more difficult to obtain/create as-built BIMs for existing buildings and industrial plants since there is no design 3D BIM model exists for these buildings, therefore an as-built BIM model would need to be built from scratch using 2D asbuilt drawings and project specifications.

Integrated BIM-FM systems offer significant benefits to owners such as better visualization of the spaces to be maintained in a virtual environment. BIMs are reviewed on a regular basis during project coordination meetings between various stakeholders, and are updated regularly during the construction phase. However, some coordination issues maybe realized within the BIM, but fixed in the field; and those changes are not typically reflected in the BIM since delivering an accurate as-built BIM model is not required as part of the project deliverables. Instead, 2D as-built drawings are produced from BIM models, manually updated to reflect the as-built conditions, and handed over to the owner. Typically, these 2D as-built drawings are not linked to any of the FM information systems such as computerized maintenance management systems (CMMS), electronic document management systems (EDMS), energy management systems (EMS), and building automation systems (BAS) [1], therefore they are not used effectively for building operations and maintenance. All the FM information systems support FM activities individually; thus the information is 
fragmented and requires manual re-entry of the data, which is labour intensive and error prone. Instead, a central BIM centric FM system would provide many benefits including locating building components easily, and facilitating real-time data access.

Today's as-built data acquisition technologies enable capturing very comprehensive and accurate as-built condition information, therefore to support BIM-FM integration. There are two major types of as-built data acquisition technologies: photo/video-grammetry (image-based) and 3D laser scanning (range-based). Photo/video-grammetry is an image based technology that involves capturing still images/video frames and processing them into 3D point clouds using computer vision techniques such as Structure from Motion (SfM). 3D Laser scanning technology, also called high definition surveying, on the other hand, is a range based technology that measures 3D coordinates of the target object/scene and produces 3D point cloud as output.

Three dimensional (3D) point clouds provide accurate and comprehensive as-built information, and are used for creating as-built BIMs. The process of creating or reconstructing as-built BIM from 3D point cloud data is called Scan-to-BIM. Currently available off-the-shelf Scan-to-BIM software packages require significant manual user input making the entire process cumbersome and error prone. Significant amount of research has been done both in academia and in industry to automate Scan-to-BIM process [3, 4, 5, 25, 26]. The contribution of this paper is a comprehensive review on currently available off-the-shelf Scan-to-BIM software and their capabilities, while identifying challenges and the direction for future research.

The following section presents detailed information on currently available as-built data acquisition technologies; photo/video-grammetry and terrestrial laser scanning respectively. The next section then provides a review of the most common readily available off-the-shelf Scan-to-BIM modeling software, which is followed by a discussion and finally conclusions including a discussion for future research.

\section{Available Technologies for As-Built Data Acquisition}

As-built data acquisition is the process of capturing the shape and structure (i.e., spatial coordinates) of an object in the point cloud format [6]. Current as-built data acquisition procedures primarily involve on-site surveys, where measurements are performed manually [7]. Such visual observations and manual surveying are the most dominant as-built data acquisition approaches, but they are time-consuming, error-prone, and infrequent, making quick and reliable decision-making difficult [8].
Recently, as-built data started being collected with two main non-contact spatial survey technologies, which are based on photo/video-grammetry (imagebased technologies) and terrestrial laser scanning (range-based technologies), in an efficient manner [9]. Advantages and drawbacks of both survey technologies are briefly presented in the next subsections.

\subsection{Photo/Video-grammetry}

Advances in photo/video-grammetry enabled accurate 3D reconstruction of civil infrastructure that is either under construction or in operation. Photo/videogrammetry adopts images or videos to derive $3 \mathrm{D}$ spatial data of the object or scene of interest [10-13]. The difference between the photo- and video-grammetry is that photogrammetry involves deriving geometric information of the object or scene of interest using information from images whereas videogrammetry uses video frames [14]. The basic principle of photo/videogrammetry is triangulation, whereby a point in space is reconstructed from two mathematically converging lines from 2D locations of the point in different images [12, 13]. Once camera calibration and orientation are computed, acquired images or video frames are used to define the geometric features of the target object or scene. In practice, this process is performed interactively by the user, who identifies corresponding geometric features in at least two images that are oriented toward each other. Some commercially available software implemented this procedure (e.g., Bundler [15] + Patch-based Multi-view Stereo [16], PhotoSynth [17] and PhotoModeler [18]). Then, the object or scene of interest can be reconstructed in 3D by using image-matching techniques.

\subsection{Terrestrial Laser Scanning}

Terrestrial laser scanning (TLS) - also called laser distance and ranging (LADAR) - enables direct acquisition of $3 \mathrm{D}$ coordinates from the surface of a target object or scene that are visible from the laser scanner's viewpoint $[11,13,19,20,21]$. TLS is based on either time-of-flight (TOF) or phase-based technology to collect range ( $\mathrm{x}, \mathrm{y}$, and $\mathrm{z}$ ) and intensity data of objects in a scene. The two technologies differ in calculating the range, while both acquire each range point in the equipment's spherical coordinate frame by mounting a laser on a pan-and-tilt unit that provides the spherical angular coordinates of the point. TOF scanners emit a pulse of laser light to the surface of the target object or scene and calculate the distance to the surface by recording the round trip time of the laser light pulse. Phase based scanners measure phase shift in a continuously emitted and returned sinusoidal wave. Both types of TLS achieve similar point measurement 
accuracies. They differ in scanning speed and maximum scanning range. Typically, phase-based TLS achieve faster data acquisition (up to one million points per second), while TOF-based TLS enables collecting data from longer ranges (up to a kilometre)

Compared to photo/video-grammetry-based survey, measurements do not require presence of distinctive and sufficient texture on the surface of the target object or scene $[11,19]$. The acquisition of $3 \mathrm{D}$ point clouds is a fully automated process in TLS [11]. Each distance measurement can be accurate to a few centimeters or less than a millimeter, depending on the sensor type, distance from the laser scanner to the surface of the target object or scene, and the characteristics of the surface being surveyed [21-23]. TLS enables collecting dense and accurate 3D point clouds in a short amount of time, and very little training is required for users. For these reasons, it is widely used in the AEC-FM industry to survey civil infrastructures [9, 24].

\section{Review of the Current off-the-shelf 3D Modelling Technologies}

Both in academia and in industry, there has been tremendous effort toward developing fully automated Scan-to-BIM (3D modeling from point clouds) algorithms. Currently available off-the-shelf 3D modeling software requires intensive manual user input, which is tedious and error prone. Several software companies have implemented semi-automated modeling tools into their Scan-to-BIM software (e.g., RealWorks developed by Trimble Navigation, Ltd., Cyclone developed by Leica Geosystems, Ag, and EdgeWise3D developed by ClearEdge3D). Their details and experimental comparisons are provided below.

\subsection{Experimental Setup}

In order to evaluate the capability of semi- or fullyautomatic modeling functions of Scan-to-BIM software, as-built MEP data were acquired using ScanStation C10 by Leica Geosystems, Ag. For this purpose, laser scanning was performed from 19 scan positions at the 12th floor of a new dormitory construction project located at Chung-Ang University campus. Following laser scan data collection, all 19 scan point clouds were registered into a common coordinate system. Figure 1(a) shows an outside view of the registered 3D point cloud, and Figure 1(b) shows an inside view of the registered 3D point cloud. An asterisk symbol indicates each scan position. Figure 2 presents the inside view of the registered 3D point cloud (section of the laser scan data that is used in the experiments) with its intensity values.

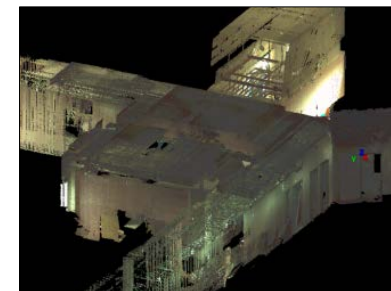

(a)

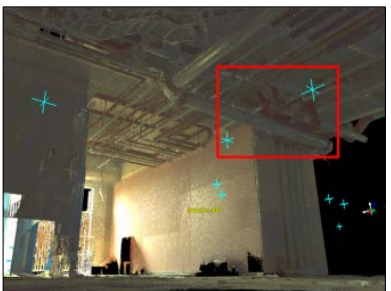

(b)
Figure 1. (a) Outside view of laser-scan data with color values; (b) Inside view of laser-scan data with color values

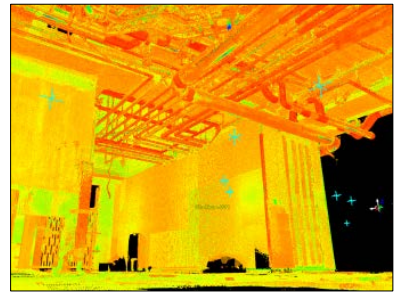

Figure 2 Inside view of laser scan data with intensity values (same view as Figure $1 \mathrm{~b}$ )

\subsection{Trimble RealWorks}

Trimble RealWorks ${ }^{\circledR}$ package is a point-cloud processing software that provides 3D modeling functions. Trimble RealWorks Advanced-Modeler ${ }^{\circledR}$ module enables modelling 3D objects from point clouds, particularly objects like columns, beams, walls, and others. In addition, Trimble RealWorks AdvancedPlant $^{\circledR}$ module is equipped with modelling functions, specifically ones related to the power, process, plant and related environments. In this study, Trimble RealWorks Advanced-Plant ${ }^{\circledR} 9.0$ was used for the evaluation.

The advanced-plant module provides users with a semi-automated function to model a pipeline, named the "EasyPipe Tool." Once a user selects a region on a pipeline surface (see Figure 3(a)), this function automatically grows regions and extracts smoothly connected surface from the selected region (see Figure 3(b)). The region selected by the user and the extracted pipeline surface are highlighted in yellow in Figure 3.

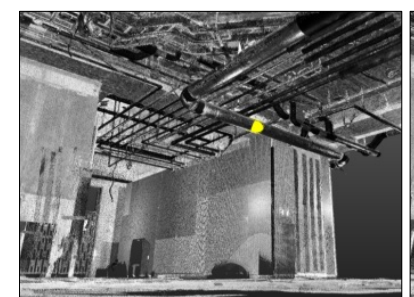

(a)

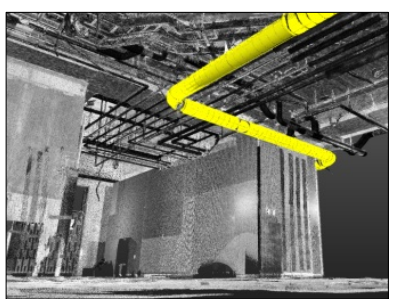

(b)
Figure 3. (a) Selected region to be modelled by user; (b) A pipeline extracted from the selected region applying automatic pipe tracking function 
Once region growing is completed, the user should then decide whether the pipeline extraction result is satisfactory or not. If the entire pipeline is extracted, then it can be modelled following the surface smoothing and geometric modelling steps (see Figure 4). Although these processes are done using a user-friendly interface, significant user intervention, which is undesirable, is needed to identify and model each pipeline.

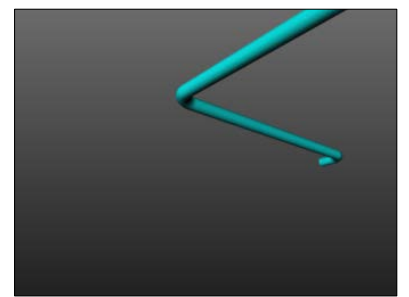

Figure 4. Pipe modelling result applying pipe modelling function

Another Trimble product that can be used to create 3D models from point clouds is Trimble SketchUp ${ }^{\circledR}$. The Trimble Scan Explorer Extension enables users to import 3D point-cloud data into Trimble SketchUp ${ }^{\circledR}$ seamlessly in order to create models from 3D scan data. Trimble SketchUp ${ }^{\circledR}$ has an automated plane extraction tool to increase modelling efficiency, particularly when modelling building interiors and facades. This software was not considered in the quantitative comparison as it was not available to the authors. However, it is included here as it is one of the current state-of-the-art as-built modelling software available in the AEC-FM market.

\subsection{Leica Cyclone}

Leica Geosystems developed software programs containing several functions that enable 3D modeling from point clouds. The latest version of Leica Cyclone ${ }^{\circledR}$ 8.1 by Leica Geosystems provides users an interface containing tools for pipe modeling with several functions, such as automatic pipe finder, region growing from the selected 3D points for cylindrical objects, cylinder fitting, and model creation from 3D point clouds. It contains various geometric types that can be used for pipe modeling; for example, cylinder, elbow, reducing elbow, cone, torus, reducer (eccentric and concentric), and pipe tee. In this study, automatic pipefinding function of Leica Cyclone ${ }^{\circledR} 8.1$ was used for the evaluation. Figure 5 shows pipe-modelling results applying the automatic pipe-finding function to the laser-scan data shown in Figures 1(b) and 2.

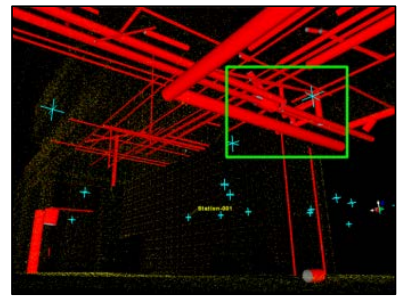

Figure 5. Pipe modelling result applying automatic pipefinding function of Leica Cyclone ${ }^{\circledR} 8.1$

Figure 6 shows a magnified portion of Fig. 4 and a corresponding photographic image. This process was completed within a few minutes when dealing with a scan point cloud containing about 70 million points. However, these types of automation tools/algorithm improvements have been limited to detecting straight portions of a given pipeline, whereas the entire pipeline includes different types of pipes, such as elbows, tees, and reducers. Hence, significant user intervention is still required in order to identify the different types of pipes (other than the straight ones).

Leica Geosystems also released several plug-in tools for pipe modeling from 3D point clouds: Leica CloudWorx AutoCAD Pro 5.0, Leica CloudWorx for Revit version 1.0.2, and Leica CloudWorx Microstation 4.0. By using these plug-in tools, it is now possible to import and process the $3 \mathrm{D}$ point clouds inside AutoCAD, Revit, and Microstation. They provide several functions for pipe modeling, such as "pipe fit" to generate cylinders based on least-squares fitting from the selected 3D point clouds and "connect pipe" to connect cylinders with elbows.

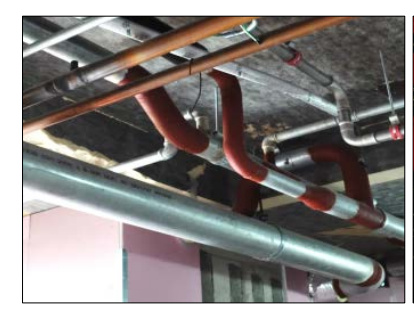

(a)

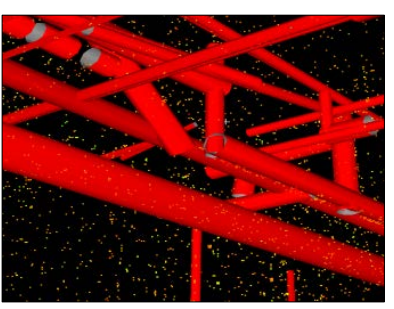

(b)
Figure 6. (a) Photographic image; (b) Magnified portion of Figure 5

\subsection{ClearEdge3D EdgeWise ${ }^{3 \mathrm{D}}$}

ClearEdge3D EdgeWise ${ }^{3 \mathrm{D}}$ Plant and BIM Suite provide a function to automatically detect the straight portions of pipelines and fit cylinders into them, named "Create a pipe model." Also, elbows and tees can be modeled by using a function named "Easy Connect.” It provides a powerful engine that can handle large 3D point clouds. In order to effectively use these functions for pipe modeling, several parameters need to be tuned, including a minimum number of points to detect a pipe 
(default value is set to 80), distance tolerance (m) (default value is set to 0.0007), and confidence level, which is needed to decide whether there is a connection between pipe fragments (default value is set to $47 \%$ ). Also, there is an option that enables to decide whether to merge adjacent pipes (default is set to merge pipes) or not.

In this study, ClearEdge3D EdgeWise ${ }^{3 \mathrm{D}}$ BIM Suite 4.5.7 was used for the evaluation. Figure 7 shows the pipe modeling result applying automatic pipe modeling and connection functions with default settings from the laser-scan data shown in Figures 1(b) and 2. This process was also completed within a few minutes when dealing with the same scan point cloud containing about 70 million points.

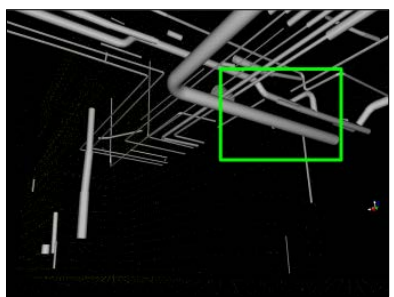

(a)

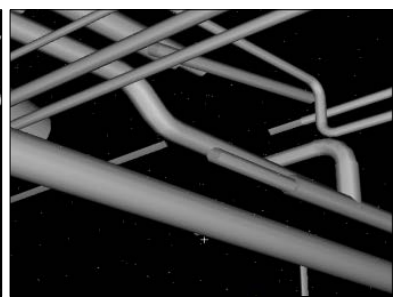

(b)
Figure 7. (a) Pipe modeling result applying automatic pipe modeling and connection functions; (b) Magnified portion of (a)

\subsection{Performance Comparisons}

This section presents performance comparison results of the "EasyPipe Tool" in Trimble RealWorks Advanced-Plant ${ }^{\circledR} 9.0$ (shown as " $A$ " in Tables 1 and 2), pipe-finding function in Leica Cyclone 8.1 (shown as " $B$ " in Tables 1 and 2), and pipe modeling and connection functions in ClearEdge3D EdgeWise ${ }^{3 \mathrm{D}}$ BIM Suite 4.5.7 (shown as "C" in Tables 1 and 2). Tables 1 and 2 summarize the automation performances for each task encountered in pipe modelling process.

Using the "EasyPipe Tool" in Trimble RealWorks Advanced-Plant ${ }^{\circledR}$ 9.0, users should identify and select a portion of the surface of each pipe in order to detect and model the entire pipeline. Although part recognition and model creation tasks can be performed automatically, applicability is limited to the straight pipes and elbows. On the other hand, entire pipelines in a given point cloud can be modelled in an automated manner by using the pipe-finding function in Leica Cyclone 8.1 and pipe modeling and connection functions in ClearEdge3D EdgeWise $^{3 \mathrm{D}}$ BIM Suite 4.5.7. However, their applicability is limited to straight pipes in Cyclone 8.1, and straight pipes and some connecting pipes such as elbows and tees in EdgeWise ${ }^{3 \mathrm{D}}$ BIM Suite 4.5.7.
Table 1. Automation performances of pipe modeling functions provided by several off-theshelf 3D modeling software

\begin{tabular}{lccc}
\hline Task & A & B & C \\
\hline Pipe Detection & $\times$ & $\circ$ & $\circ$ \\
Part Recognition & $\circ$ & N/A & $\circ$ \\
Model Creation & $\circ$ & $\circ$ & $\circ$ \\
\hline
\end{tabular}

Note: Each of the symbols $\times$ and $\circ$ indicates that $a$ function can be used in semi-automated or fullyautomated manners.

Table 2. Applicability of pipe modeling functions provided by several off-the-shelf 3D modeling software

\begin{tabular}{lccc} 
& A & B & C \\
\hline Straight pipe & $\circ$ & $\circ$ & 0 \\
Elbow & $\circ$ & $\times$ & $\circ$ \\
Tees & $\times$ & $\times$ & $\circ$ \\
\hline
\end{tabular}

Note. Each of the simbols $x$ and $\circ$ indicates that $a$ function is applicable to a type of pipe (e.g., straight pipe, elbow, or tee).

This study also evaluated the accuracy of the automatic pipe modeling functions of Leica Cyclone 8.1 and ClearEdge3D EdgeWise ${ }^{3 \mathrm{D}}$ BIM Suite 4.5.7. For this purpose, three performance measures of precision, recall, and error values were considered.

- Precision is indicated by the ratio of the number of modeled true pipes to the total number of correctly and incorrectly modeled pipes.

- Recall is indicated the ratio of the number of modeled true pipes to the total number of true pipes.

- Error refers to the ratio of the number of incorrectly modeled pipes to the total number of true pipes.

Modeling software that perform better in detecting and modeling more true pipes should have higher precision and recall values, while having a lower error value. Summary statistics of the three performance measures for each pipe modeling function of each offthe-shelf 3D modeling software program are provided in Tables 3 and 4.

In summary, the results indicate that user intervention is required to identify whether the modeled pipes are correctly fitted and to uncover any undetected portions that need to be modeled. Using the pipe-finding function in Leica Cyclone 8.1, 57 out of 78 straight pipes to be modeled were modeled and the remaining 21 pipes were not modeled. However, among 57 straight pipes, 15 pipes were modeled with larger or smaller pipes compared to the true sizes (radius). Also, most of the 57 straight pipes' axes were slightly different and shifted from the as-built pipes' axes, and most of their lengths were longer or shorter than the true lengths between their flanges. These cases were not considered 
as incorrectly modeled pipes. Here, the pipes that were modeled but do not exist were counted as incorrectly modeled pipes. Moreover, 49 cylinders were unnecessarily fitted to the walls, h-beams, electrical cable ducting, and other objects.

Table 3. Evaluation result of pipe modeling function provided in Leica Cyclone 8.1

\begin{tabular}{lrrr}
\hline & Precision & Recall & \multicolumn{1}{c}{ Error } \\
\hline Straight Pipes & $42 / 127$ & $42 / 78$ & $85 / 78$ \\
& $(33.1 \%)$ & $(53.8 \%)$ & $(109.0 \%)$ \\
Connecting Pipes & & & \\
Elbows & N/A & N/A & N/A \\
Tees & N/A & N/A & N/A \\
Reducers & N/A & N/A & N/A \\
\hline
\end{tabular}

Using the pipe modeling and connection functions in ClearEdge3D EdgeWise ${ }^{3 \mathrm{D}}$ BIM Suite 4.5.7, 59 out of 78 straight pipes were modeled, and the remaining 19 pipes were not modeled. Among 59 straight pipes, 5 pipes were modeled with larger or smaller pipes compared to the true sizes (radius). Also, most of the 59 straight pipes' lengths were longer or shorter than the true lengths. Moreover, 30 cylinders were unnecessarily fitted to the walls, h-beams, electrical cable ducting, and other objects. For elbows, 13 out of 44 elbows were modeled, and the remaining 31 elbows were not modeled. Among 13 elbows, one elbow was modeled with a smaller pipe compared to the true size (radius). Moreover, 6 elbows were unnecessarily fitted. For tees, only 1 out of 5 tees were correctly modeled, while the remaining 4 tees were not modeled.

Table 4. Evaluation result of pipe modeling function provided in ClearEdge3D EdgeWise3D BIM Suite 4.5.7

\begin{tabular}{lrrr}
\hline & Precision & Recall & \multicolumn{1}{c}{ Error } \\
\hline Straight Pipes & $54 / 108$ & $54 / 78$ & $54 / 78$ \\
& $(50.0 \%)$ & $(69.2 \%)$ & $(69.2 \%)$ \\
Connecting Pipes & & & \\
Elbows & $12 / 50$ & $12 / 44$ & $38 / 44$ \\
& $(24.0 \%)$ & $(27.3 \%)$ & $(86.4 \%)$ \\
Tees & $1 / 5$ & $1 / 5$ & $4 / 5$ \\
& $(20.0 \%)$ & $(20.0 \%)$ & $(80.0 \%)$ \\
Reducers & N/A & N/A & N/A \\
\hline
\end{tabular}

\section{Conclusions}

This paper presented a comprehensive review and an evaluation of the currently available off-the-shelf Scanto-BIM (3D modeling/object detection from 3D point clouds) software through a set of experiments in terms of their level of automation in detecting pipelines. Real life data collected from a building construction site at Chung-Ang University campus was used for the experiments. The experimental results show that the all three software evaluated facilitate a semi-automated 3D modeling process, i.e. they still require significant user intervention to identify whether the modeled pipes fit correctly or to uncover any undetected portions such as elbows that need to be modeled. Semi-automated modeling tools may be sufficient for modeling small mechanical rooms; but not for modeling large complex industrial plants.

Simultaneously, several researchers also studied 3D object reconstruction, specifically for MEP components, from laser scan point clouds to achieve a fully automated Scan-to-BIM process. Specifically the method proposed in [5], which integrates automated Hough transform and Scan-vs-BIM [27] algorithms, is very promising as it facilitates very accurate MEP object reconstruction. The future research should focus on integrating existing object recognition and 3D modeling techniques, along with developing new algorithms to achieve a fully automated Scan-to-BIM process.

Accurate as-built BIM models are necessary for FM activities. The possible benefits of using BIM during FM stage are widely acknowledged; however their implementation does not go beyond a few case studies. One of the main reasons for this is that as-built BIM models are not asked by the owners; therefore they are not part of the contract document submittals. Design BIM models are typically updated during the construction stage, but some coordination issues maybe realized within the model, but fixed in the field. And those changes are not typically reflected in the BIM model. Any progress made toward automated Scan-toBIM process will help enable efficient BIM-FM integration, which would translate into significant cost savings during facility operations and maintenance phase.

\section{Acknowledgments}

This research was supported by Basic Science Research Program through the National Research Foundation of Korea (NRF) funded by the Ministry of Education (NRF-2013R1A1A2A10058175).

\section{References}

[1] Becerik-Gerber B., Jazizadeh F., Li N., and Calis G. Application areas and data requirements for BIM-enabled facilities management, J. Constr. Eng. Manage., 138(3): 431-442, 2012.

[2] Bryde D., Broquetas M., and Volm J.M. The project benefits of building information modelling (BIM). Int. J. Proj. Manag., 31(7): 971-980, 2013. 
[3] Hajian H. and Becerik-Gerber B. Scan to BIM: factors affecting operational and computational errors and productivity loss. In Proceedings of the 27th International Symposium on Automation and Robotics in Construction (ISARC), pages 265-272, Bratislava, Slovakia, 2010.

[4] Adan A., Xiong X., Akinci B., Huber D. Automatic creation of semantically rich 3D building models from laser scanner data. In Proceedings of the 28th International Symposium on Automation and Robotics in Construction (ISARC), pages 342-347, Seoul, Korea, 2011.

[5] Bosché F., Ahmed M., Turkan Y., Haas C. T., and Haas, R. The value of integrating Scan-to-BIM and Scan-vs-BIM techniques for construction monitoring using laser scanning and BIM: The case of cylindrical MEP components. Automat. Constr., 49(Part B): 201-213, 2015.

[6] Dai F., Rashidi A., Brilakis I., and Vela P. Comparison of image-based and time-of-flightbased technologies for three-dimensional reconstruction of infrastructure, J. Constr. Eng. Manage., 139(1): 69-79, 2013.

[7] Klein L., Li N. and Becerik-Gerber B. Imagedbased verification of as-built documentation of operational buildings, Automat. Constr., 21: 161171, 2012

[8] Golparvar-Fard M., Peña-Mora F., Savarese S. Integrated sequential as-built and as-planned representation with D4AR tools in support of decision-making tasks in the AEC/FM industry, $J$. Constr. Eng. Manage., 137(12): 1099-1116, 2011 a.

[9] Volk R., Stengel J., and Schultmann F. Building Information Modeling (BIM) for existing buildings - Literature review and future needs, Automat. Constr., 38: 109-127, 2014.

[10] Luhmann T., Robson S., Kyle S., and Harley I. Close Range Photogrammetry: Principles, Techniques and Applications, first ed. John Wiley \& Sons, New York, NY, 2006.

[11] Alba M.I., Barazzetti L., Scaioni M. Rosina E., and Previtali M. Mapping infrared data on terrestrial laser scanning 3D models of buildings, Remote Sensing, 3(9): 1847-1870, 2011.

[12] Fathi H. and Brilakis I. Automated sparse 3D point cloud generation of infrastructure using its distinctive visual features, Adv. Eng. Inform., 25(4): 760-770, 2011.
[13] Golparvar-Fard M., Bohn J., Teizer J., Savarese S., and Peña-Mora F. Evaluation of image-based modelling and laser scanning accuracy for emerging automated performance monitoring techniques, Automat. Constr., 20(8): 1143-1155, 2011b.

[14] Bhatla A., Choe S.Y., Fierro O., and Leite F. Evaluation of accuracy of as-built 3D modelling from photos taken by handheld digital cameras, Automat. Constr., 28: 116-127, 2012.

[15] Snavely N. Bundler: Structure from motion (SfM) for unordered image collections. Online: http://phototour.cs.washington.edu/bundler, Accessed:15/01/2015

[16] Furukawa Y. and Ponce J. Accurate, dense, and robust multiview stereopsis, IEEE Transactions on Pattern Analysis in Machine Intelligence. 32(8): 1362-1376, 2010.

[17] Microsoft Corporation: Photosynth. Online: http://photosynth.net/, Accessed: 20/01/2015

[18] Eos Systems, Inc.: PhotoModeler. Online: http://www.photomodeler.com/index.htm, Accessed: 03/01/2015

[19] Kiziltas S., Akinci B., Ergen E., Tang P., and Gordon C. Technological assessment and process implications of field data capture technologies for construction and facility/infrastructure management, ITcon., 13: 134-154, 2008.

[20] Vosselman G. and Maas H.-G. Airborne and Terrestrial Laser Scanning, first ed. Whittles Publishing, Dunbeath, UK, 2010.

[21] Xiong X., Adan A., Akinci B., and Huber D. Automatic creation of semantically rich 3D building models from laser scanner data, Automat. Constr., 31: 325-337, 2013.

[22] Jaselskis E., Gao Z., and Walters R.C. Improving transportation projects using laser scanning, $J$. Constr. Eng. Manage., 131(3): 377-384, 2005.

[23] Arayici Y. An approach for real world data modelling with the 3D terrestrial laser scanner for built environment, Automat. Constr., 16(6): 816829, 2007.

[24] Tang P. and Akinci B. Automatic execution of workflows on laser-scanned data for extracting bridge surveying goals, Adv. Eng. Inform., 26(4): 889-903, 2012. 
[25] Pătrăucean V., Armeni I., Nahangi M., Yeung J., Brilakis I., and Haas C. State of research in automatic as-built modelling, Advanced Engineering Informatics, (in press), 2015.

[26] Son H., Kim C., and Kim C. 3D reconstruction of as-built industrial instrumentation models from laser-scan data and a 3D CAD database based on prior knowledge, Automation in Construction, 49, 193-200, 2015.

[27] Bosche, F., \& Haas, C. T. (2008). Automated retrieval of 3D CAD model objects in construction range images. Automation in Construction, 17(4), 499-512. 